\title{
Über die Mitoseverhältnisse im Felchenei (Coregonus lavaretus L. Steinmann) $^{1}$
}

\author{
Von H. A мв
}

(Aus der Eidgenössischen Anstalt für Wasserversorgung, Abwasserreinigung und Gewässerschutz an der Eidgenössischen Technischen Hochschule in Zürich).

Eingegangen am 16. März I953

\section{EINLEITUNG}

Im Jahre I945 veröffentlichte G. SvAERDSON eine umfangreiche Arbeit über die Chromosomenverhältnisse der Salmoniden, die er an wenige Stunden bis Tage alten befruchteten Eiern untersucht hatte. Dabei kamen eine ganze Reihe wichtiger zytologischer Ergebnisse heraus:

Der Autor fand als diploiden Chromosomensatz für Coregomus lavaretus und für C. albula (schwedische Formen!) die Zahl 80. Es gelang ihm dabei, die analysierten Sätze in Gruppen von je vier in bezug auf Grösse und Form gleichwertiger Chromosomen aufzuteilen. In allen untersuchten Felchenformen fand SvaERdSON Eier mit Mitosestörungen, ähnlich denjenigen, die er bei Rückkreuzungsbastarden finden konnte. Dazu treten stets - in jedem Verhältnis mit normalen Kernen gemischt - Kerne mit verschiedener Chromosomenzahl auf, wobei am häufigsten Zahlen um 20, 40 und 60 vorkommen. Als kleinste Chromosomenzahl fand er 4 , enthalten als Satz eines in Mitose befindlichen Kernes.

Die Tatsache, dass neben 80 (diploide Chromosomenzahl) am häufigsten 20, 40 und 60 Chromosomen auftreten, deutet SvaERDson dahin, dass die Koregonen (bzw. die Salmoniden im allgemeinen) alte Polyploide sind.

E. KUPKA (I948) bearbeitete mehrere Felchenrassen des Zürich- und des Vierwaldstättersees. Er stellte eine diploide Zahl von 72 Chromosomen

1) Vorläufige Mitteilung. 
fest und konnte - wie schon SVAERDSON - aus den analysierten Sätzen Vierergruppen bilden. Daneben ermittelte er haploide Felchenformen mit 36 Chromosomen, deren Sätze bezeichnenderweise bloss eine Aufteilung in Zweiergruppen zuliessen. Auch gelang es KuPKA, einzelne Felchenformen durch rein zytologische Merkmale zu unterscheiden, indem er das Vorhandensein von längeren oder kürzeren SAT-Chromosomen als Kriterium bewertete.

In einer späteren Arbeit (1950) über Laichmaterial von Atterseefelchen berichtet KUPKa über das Auftreten einzelner Embryonen mit durchwegs I8 Chromosomen. Dieser «hemihaploide» Satz (n/2) lässt sich nicht in Gruppen aufteilen.

Diese bedeutsame Tatsache lässt sich nur durch die Annahme erklären, dass der haploide Satz des Atterseefelchens bereits aus zwei verschiedenen Teilsätzen aufgebaut ist. Eine weitere Beobachtung stützt diesen Erklärungsversuch: Die erwähnten Vierergruppen, die sich in der Regel aus diploiden Sätzen bilden lassen, können weiter in Zweiergruppen unterteilt werden, die sich untereinander wohl sehr ähnlich, nicht aber identisch sind. Das Auftreten von Phasenverscbiedenbeiten im zeitlichen Ablauf der Mitose, woran eine Hälfte des ganzen Satzes beteiligt ist, weist darauf hin, dass sich die Chromosomensätze in je zwei gleich grosse Teilgenome trennen lassen, wobei je eine Zweiergruppe (entstanden durch Teilung einer Vierergruppe) dem einen, die andere dem andern Teilgenom zukommt.

Aus diesen Feststellungen erhellt, dass die Entstehung des AtterseeBlaufelchens einer allopolyploiden Chromosomenkombination zuzuschreiben ist.

KUPKA nimmt als wahrscheinlich an, dass bei den Felchen drei ursprüngliche Sätze vorhanden seien, welche bei den verschiedenen Rassen verschieden miteinander kombiniert wurden. Damit soll auch die grosse Schwierigkeit in der Systematik der Koregonen (STEINMANN, I95I) verständlich werden, da die Allopolyploidie zur Überschneidung morphologischer Merkmale führen kann.

\section{NEUE UNTERSUCHUNGEN}

\section{Material, Methode}

Für unsere Untersuchungen verwendeten wir die bei KuPKA (1950) beschriebene Orcein-Essigsäure-Quetschmethode; für die mikroskopische Analyse wurde das Phasenkontrastverfahren verwendet. 
An Eimaterial wurde untersucht:

Zürichsee: Schwebfelchen (Stäfa); «Albeli» (Stäfa); Blalig (Rapperswil); Zürcher Obersee: Albeli (Bollingen);

Untersee: Blaufelchen (Ermatingen); Gangfisch (Ermatingen).

Sämtlicher Laich wurde in der Laichzeit I950/5I von den zuständigen Fischereiorganen auf die übliche bewährte Art gewonnen, auf schonendste Weise ins Labor verbracht und dort in Zugergläsern gehältert.

Aus insgesamt II8 Embryonen (Keimscheiben im Alter von 24 bis I02 Stunden) wurden an die I60 Metaphaseplatten photographiert, soweit als möglich unter dem Mikroskop ausgezählt und über 90 nach dem direkten mikroskopischen Bild unter Zuhilfenahme der Mikrophotos ausgezeichnet und damit genau ausgezählt. Dazu wurden eine ganze Reihe von Platten analysiert.

Die vorliegende Arbeit verfolgte ursprünglich den Zweck, die Resultate von KUPKA kurz nachzuprüfen; dass sie grösser wurde, als beabsichtigt, liegt in der Art der Ergebnisse begründet.

\section{A. Die Chromosomenzahl}

\section{von Coregonus lavaretus L. (Steinmann)}

Im Einklang mit SvaERDSON fanden wir nicht eine oder zwei, sondern eine ganze Reihe verschiedener Chromosomenzahlen. SvAERDSON traf dieses Phänomen hauptsächlich bei kältebehandelten Eiern an; unser Laichmaterial dagegen wurde in keiner Weise künstlich behandelt.

In 'Tabelle I sind die ausgezählten Metaphaseplatten nach ihrer Anzahl der Chromosomen zusammengestellt. Es handelt sich dabei kaum um statistisch verwendbare Werte, sondern um Platten, die lediglich ihrer Klarheit wegen herausgegriffen wurden. Bei der Untersuchung war jeweils nicht die «interessante» Chromosomenzahl, sondern ausschliesslich die Klarheit in der Anordnung der Chromosomen für die Auswahl massgebend. Es ist schlechterdings unmöglich, sämtliche Metaphaseplatten eines Embryos auszuzählen, da nur die wenigsten genügend klar sind, dass sich die Auszählung überhaupt rechtfertigt. Sobald eine Platte nicht mehr ausgezeichnet werden kann, ist sie auch für eine Zählung nurmehr bedingt brauchbar. In der Laichzeit 1952/53 wurde dagegen für statistische Zwecke neues Material präpariert, doch mussten wir die Verarbeitung noch zuriickstellen. Eine kurze Durchmusterung zeigte indessen dieselben prinzipiellen Verhältnisse wie in Tabelle $I$. 
Tabelle 1

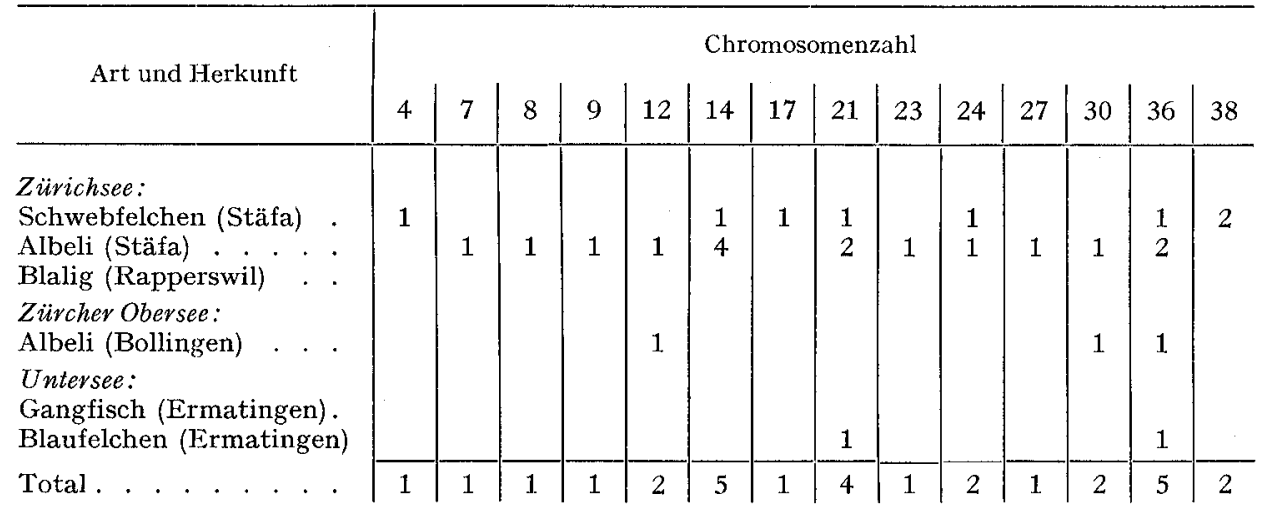

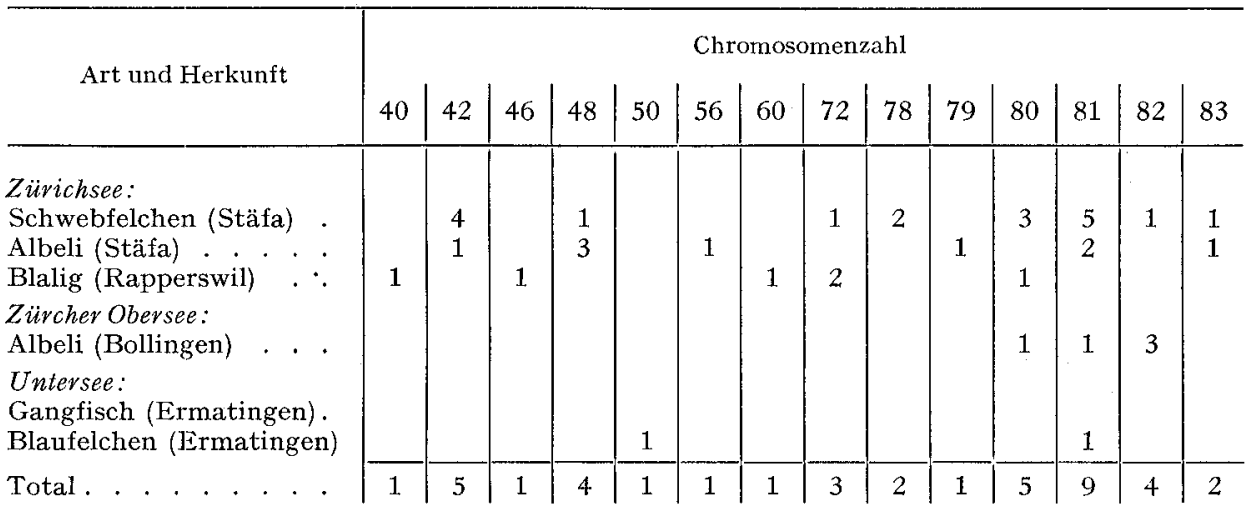

\begin{tabular}{|c|c|c|c|c|c|c|c|c|c|c|c|c|}
\hline \multirow{2}{*}{ Art und Herkunft } & \multicolumn{12}{|c|}{ Chromosomenzahl } \\
\hline & 84 & 85 & 86 & 87 & 88 & 90 & 91 & 92 & 146 & $150 \pm 2$ & $|165+10|$ & $590 \pm 10$ \\
\hline $\begin{array}{l}\text { Zürichsee: } \\
\text { Schwebfelchen (Stäfa) . } \\
\text { Albeli (Stäfa) . } \cdot . \\
\text { Blalig (Rapperswil) } .\end{array}$ & $\begin{array}{l}8 \\
3 \\
2\end{array}$ & 1 & 1 & 1 & 1 & $\begin{array}{l}2 \\
1 \\
3\end{array}$ & 1 & & 1 & 1 & & 1 \\
\hline $\begin{array}{l}\text { Zürcher Obersee: } \\
\text { Albeli (Bollingen) }\end{array}$ & 6 & & & & 1 & 1 & & & & & & \\
\hline $\begin{array}{l}\text { Untersee: } \\
\text { Gangfisch (Ermatingen). } \\
\text { Blaufelchen (Ermatingen) }\end{array}$ & $\begin{array}{l}1 \\
7 \\
\end{array}$ & 2 & & & $\begin{array}{l}2 \\
1 \\
\end{array}$ & & & 1 & & & 1 & \\
\hline Total . . . . . . . & 27 & 3 & 1 & 1 & 5 & 7 & 1 & 1 & 1 & 1 & 1 & 1 \\
\hline
\end{tabular}


Immerhin dürfen wir bei gleicher Behandlung aller Embryonen eine gewisse Wahrscheinlichkeit dafür erwarten, dass man von jeder Chromosomenzahl verhältnismässig ungefähr gleich viel Exemplare «erwischt». Die Schwankungen dieses "ungefähr» können indessen erst an Hand eines grösseren Materials ermittelt werden.

In Tabelle I finden wir (die erwähnte Voraussetzung vorbehalten!), dass bestimmte Zahlen hervortreten:

$$
\text { I4; } 2 \text { I } 36 ; 42 ; 48 ; 72 ; 8 \mathrm{I} ; 84 ; 88 ; 90 .
$$

Diese Zahlen, wenigstens 84,72 und darunter, stehen untereinander in einem bestimmten Verhältnis, indem sie entweder der Siebner- oder der Zwölferreihe angehören. Wir haben hier also ein ähnliches Bild vor uns, wie es bereits SVAERDSON bei schwedischen Felchen gefunden hat, immerhin mit dem Unterschied, dass sich seine Chromosomenzahlen in einer Zehnerreihe bewegen.

Es konnte in keinem Fall festgestellt werden, dass ein Embryo bloss Kerne mit ein und derselben Chromosomenzahl besitzt (vgl. KupKA, I950), sondern wir haben es mit « unbalanced embryos» (SVAERDSON) zu tun. Einige Beispiele mögen dies illustrieren:

Schwebfelchen (Stäfa):

I4; $42 ; 17 ; 72$;

$42 ; 146 ; 8 \mathrm{I} ; 78$;

$36 ; 38 ; 38 ; 42 ; 90$.

Albeli (Stäfa):

I5O $\pm 2 ; 85 ; 79 ; 7 ;$

$92 ; 56 ; 48 ; 48 ; 36 ; 24 ; 24 ; 21 ; 14 ; 14 ; 14 ;$

$84 ; 8 \mathrm{I} ; 42 ; 2 \mathrm{I} ; \mathrm{I} 4 ; 9 ; 8$.

Albeli (Bollingen):

$84 ; 87 ; 84 ; \mathrm{I} 2 ; 84$.

Blaufelchen (Ermatingen):

$36 ; 21 ; 48 ; 165 \pm$ 10.

Wir sind auch ausserstande, für eine bestimmte Felchenform einen bestimmten Habitus des Chromosomensatzes anzugeben. Wie die Abbildungen I und 2 zeigen, weichen die einzelnen Sätze habitusmässig stark voneinander $a b$, handle es sich nun um 84 oder um eine andere Anzahl Chromosomen. Mit Hinsicht darauf zählten wir sämtliche in Tabelle I angeführten Metaphaseplatten detailliert aus und unterschieden dabei stabförmige, V-förmige, schleifenförmige und SAT-Chromosomen. Das 


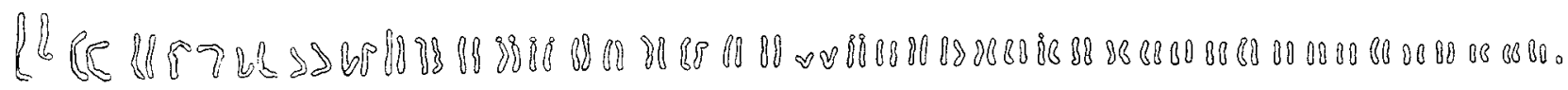

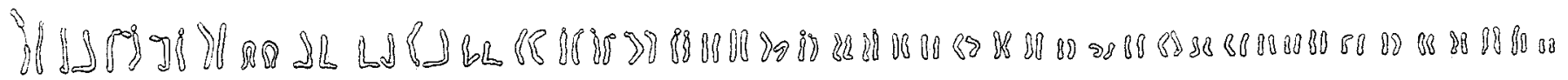

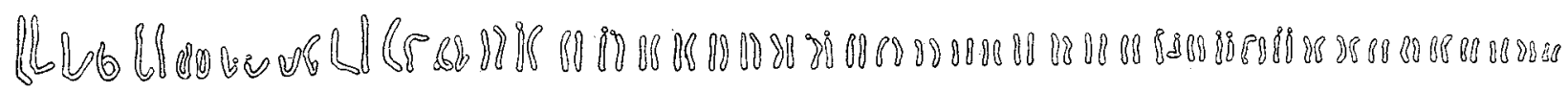

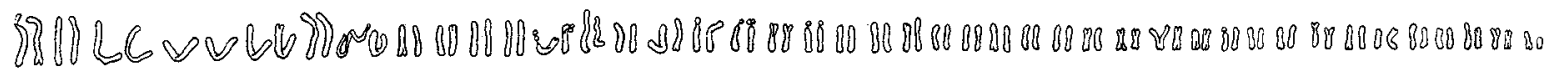

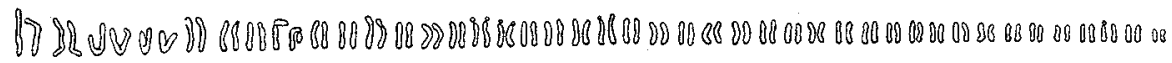

$10 \mu$

Abb. 1

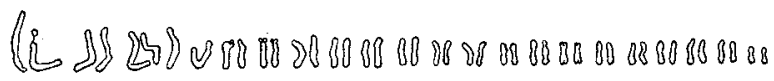

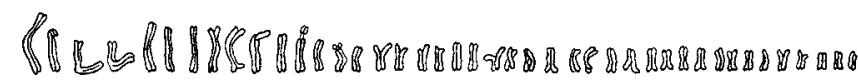

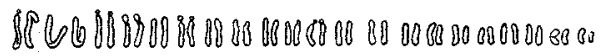

Abb. 2

$\stackrel{10 \mu}{\longrightarrow}$

Abb. 1. Schwebfelchen (Stäfa). Fünf nach Grösse und Form geordnete Sätze von je 84 Chromosomen. Der zweite und dritte Satz stammen aus demselben Präparat.

Abb. 2. Schwebfelchen (Stäfa). Drei nach Grösse und Form geordnete Sätze von je 42 Chromosomen. Der Satz rechts stammt aus dem bei Abbildung 1 erwähnten Präparat. 
Ergebnis war, dass sich auch hier keinerlei Übereinstimmung zwischen einzelnen, gleich grossen Sätzen ergab. Sogar die Anzahl und Grösse der SAT-Chromosomen, von KUPKA als Merkmal verwendet, schwankt stark (Abb. I). Wieweit die Unzulänglichkeiten der Quetschmethode dafür verantwortlich zu machen sind, vermögen wir noch nicht zu ermitteln, doch hoffen wir, dass diese Frage durch Untersuchungen nach der Mikrotomtechnik abgeklärt werden kann.

\section{B. Phasenverschiedenheiten}

Es gelang KuPKa (I95I), wie bereits in der Einleitung erwähnt, eine Metaphaseplatte, in welcher Phasenverschiedenheiten zwischen einzelnen Chromosomen feststellbar waren, zu analysieren, wobei die Hälfte aller Chromosomen (36) das normale Bild einer mittleren Metaphase, die übrigen 36 bereits einen deutlichen anaphasischen Längsspalt zeigten. Je zwei Chromosomen dieser beiden phasenverschobenen Teilsätze entsprachen einander. Es konnten also Vierergruppen zusammengestellt werden, deren eine Hälfte vom einen, die andere vom andern phasenverschobenen Halbsatz stammten, mit einer Ausnahme allerdings, indem die zwei längsten Chromosomen des einen mit zwei SAT-Chromosomen des andern Halbsatzes korrespondierten. Die Zusammenstellung jedes zweiten Chromosoms aus beiden Halbsätzen müsste also zwei Reihen von je 36 Chromosomen ergeben, die dem väterlichen und mütterlichen Satz entsprechen.

Es gelang uns ebenfalls, solche Phasenverschiedenheiten nachzuweisen, doch weichen unsere Resultate in zahlenmässiger Hinsicht von denjenigen KUPKAS ab.

Es ist nicht leicht, eine Phasenverschiebung in einer Metaphaseplatte zu beurteilen, da die Unterschiede nur in den wenigsten Fällen genügend zur Geltung kommen. Es scheint indessen, dass ein beachtlicher Teil sämtlicher Metaphasen phasenverschobene Chromosomen besitzt, doch lassen sich nur die wenigsten klar erfassen. Die folgende Zusammenstellung enthält diejenigen phasenverschobenen Metaphasen, die sich aus unserem Material klar ermitteln lassen:

\begin{tabular}{|c|c|c|}
\hline \multirow[b]{2}{*}{ Albeli (Stäfa): } & Chromosomen & Davon verschoben \\
\hline & $150 \pm 2$ & $2 \mathrm{I}-22$ \\
\hline Blaufelchen (Ermatingen): & 84 & I4 \\
\hline & 84 & $2 \mathrm{I}$ \\
\hline Albeli (Bollingen): & 84 & I4 \\
\hline
\end{tabular}


Ein Vergleich von Tabelle I mit der obigen Darstellung zeigt, dass hier wie dort rein zahlenmässig ähnliche Verhältnisse vorliegen. Diejenigen Zahlen, die für phasenverschobene Chromosomen ermittelt wurden, finden sich ebenfalls als Totalzahlen kleiner Kerne.

Phasenverschiebungen kommen nicht nur in Sätzen mit 84 Chromosomen vor. Einerseits zeigt bereits die obige Liste, dass auch Sätze von mehr als 84 Chromosomen dieses Phänomen ausbilden können, und anderseits wurde diese Erscheinung auch in kleineren Sätzen als 84 (beispielsweise 42 und 36 ) beobachtet, doch musste dort vorderhand auf eine Analyse verzichtet werden.

\section{Mehrpolige Spindeln}

SVAerdSON beobachtete an Embryonen, die tiefen Temperaturen ausgesetzt waren (cold treatment), das Auftreten multipolarer Anaphasen. Es dürfte sich dabei, worauf übrigens auch ein Bild in der betreffenden Originalarbeit hinweist, um eine abnorme Erscheinung handeln.

In unserem Material fanden wir verschiedentlich tripolare Spindeln, doch müssen wir annehmen, dass es sich nicht um eine künstlich hervorgerufene Erscheinung handelt, wie wir bereits auf S. 223 bemerkten.

Figur $S$ (Tafel I) zeigt eine solche tripolare Anaphase. Diese Erscheinung tritt recht selten auf, kann sie doch pro Embryo bloss ein- bis höchstens dreimal beobachtet werden (Kerninhalt eines Embryos von $72 \mathrm{~h}$ Bebrütungsdauer rund to00o!). Eine Auszählung ist in den wenigsten Fällen mit Sicherheit durchführbar. Immerhin konnten wir drei tripolare Anaphasebilder analysieren:

$$
\begin{aligned}
& 84 ; 42 ; 42 \pm 2 \text { Chromosomen (Tafel I, Figur 5); } \\
& 84 ; 36 ; 48 \text { (alle } \pm \text { I) Chromosomen; } \\
& 72 ; 48 ; 24 \pm \text { I Chromosomen. }
\end{aligned}
$$

Der Einwand, es könnte sich bei diesen dreipoligen Anaphasen um durch Quetschung hervorgerufene Artefakte handeln, ist in gewissem Sinne berechtigt, doch weisen die obigen Zahlen sowie die recht eindeutigen Bilder die Annahme eines Kunstproduktes eigentlich von sich. Um die Frage weiter $\mathrm{zu}$ verfolgen, sind ebenfalls Untersuchungen nach der Schneidetechnik vorgesehen. 

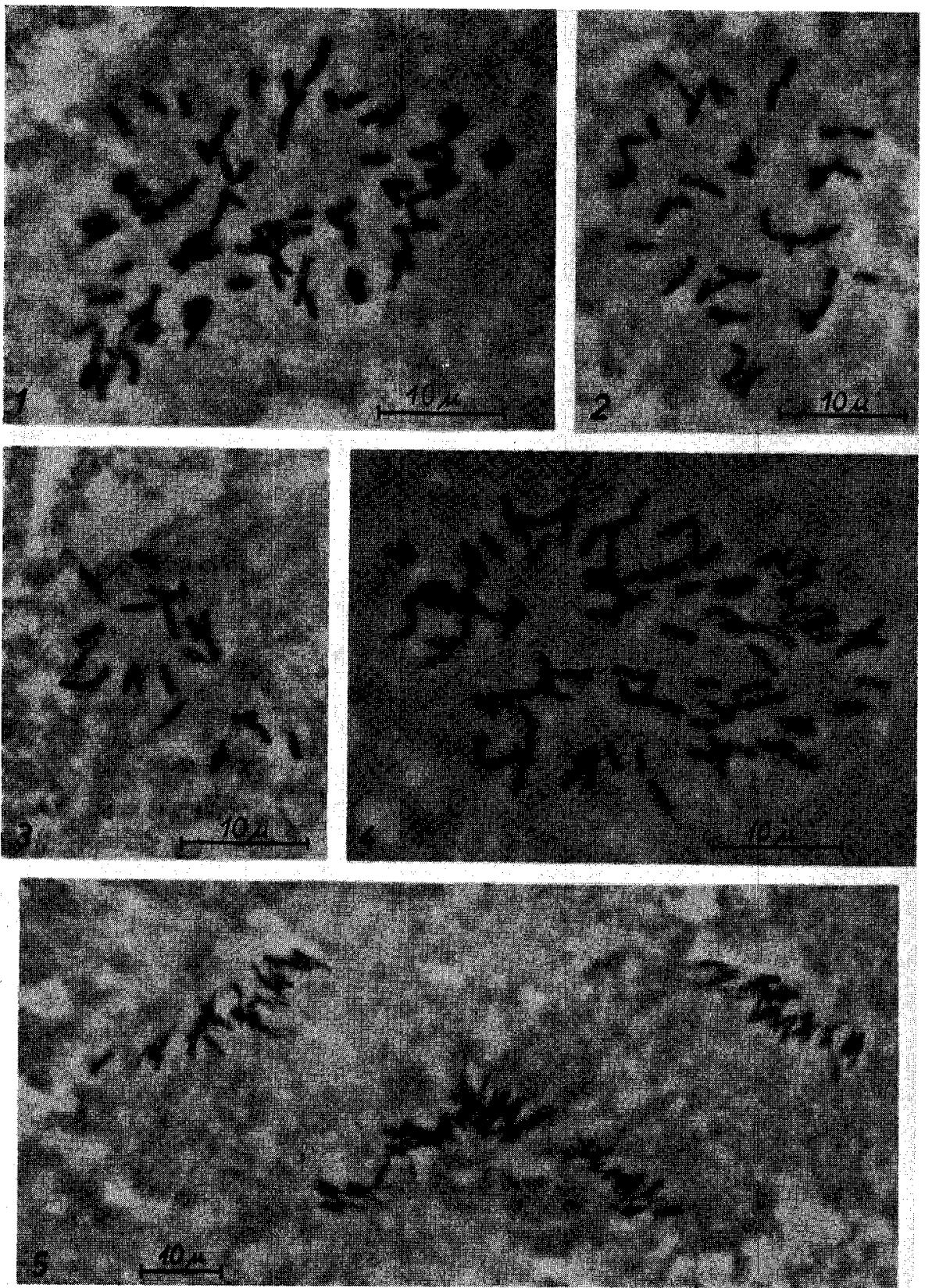

Tafel 1

Fig. 1. Albeli (Stäfa), Metaphaseplatte mit 84 Chromosomen (= entsprechender Satz aus Abb. 3).

Fig. 2. Albeli (Stäfa), Metaphaseplatte mit 42 Chromosomen (= entsprechender Satz aus Abb. 3).

Fig. 3. Albeli (Stäfa), Metaphaseplatte mit 21 Chromosomen (= entsprechender Satz aus Abb. 3).

Fig. 4. Albeli (Stäfa), Metaphaseplatte mit rund 84 Chromosomen. Die Phasenverschiebung ist deutlich erkennbar.

Fig. 5. Blaufelchen (Ermatingen). Tripolare Anaphase mit 84, 42 und $42 \pm 2$ Chromosomen. Aufnahme mit Leitz-A pochromat $40: 1$, A 0,70 (Phasenkontrastaufnahmen). 


\section{Diskussion}

Die vorliegenden Ergebnisse können wohl, wenigstens in gewissem Sinne, die Resultate von SvaERdson und von KupKa bestätigen, kaum aber die Interpretationen des letzten Autors. Wir behalten uns vor, dazu in einer späteren Mitteilung Stellung zu nehmen und verzichten vorderhand auf weitere Ausserungen, da erst noch vermehrte Resultate abzuwarten sind.

Eines dürfte jedoch zur Diskussion gestellt werden: Wie haben wir uns die Entstehung der verschiedenen Chromosomenzahlen zu denken? Handelt es sich um eine endomitotische Polyploidisierung, oder muss ein anderer, unbekannter Mechanismus gesucht werden?

Dass eine Polyploidisierung, wenigstens in Kernen von 84 oder weniger Chromosomen, nicht denkbar ist, geht aus den Abbildungen I bis 3 hervor. Obwohl SVaERdSON und KUPKa in diploiden Sätzen ( 80 bzw. 72 Chromosomen) Vierergruppen bilden können, gelingt es nach Abbildung $\mathbf{I}$ (Sätze von 84 Chromosomen) kaum, eine solche Gruppierung ebenfalls

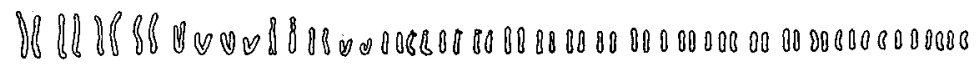

00000000080000000900800000

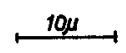

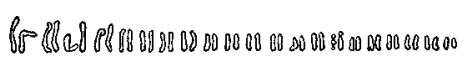

42

200. 00. 8000000080888008.

21

Abb. 3

Abb. 3. Drei Sätze von 84, 42 und 21 Chromosomen, alle aus dem gleichen Präparat. (Die entsprechenden Platten sind auf Tafel 1, Figuren 2, 3 und 4, dargestellt.) Weitere aus diesem Präparat stammende Zahlen: $9 ; 14 ; 8 ; 81$.

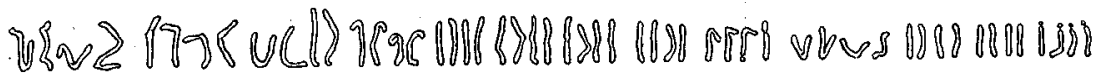

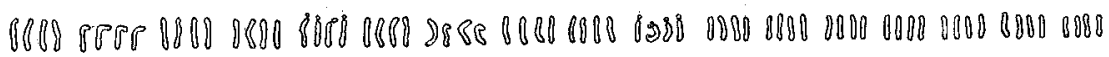

03838088008008888080800800

Abb. 4

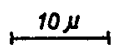

Abb. 4. Schwebfelchen (Stäfa). Platte mit 146 Chromosomen (Tafel 2, Figur 6). Nach Form und Grösse geordnet. 
vorzunehmen. Wir möchten in diesem Zusammenhang auf die Abbildungen von KUPKA (I948) hinweisen, wo Vierergruppen zusammengestellt wurden. Leider scheint diese Anordnung nicht unbedingt zu überzeugen; wir halten eher dafür, es seien dort Zweiergruppen - diese dafür sicher! -
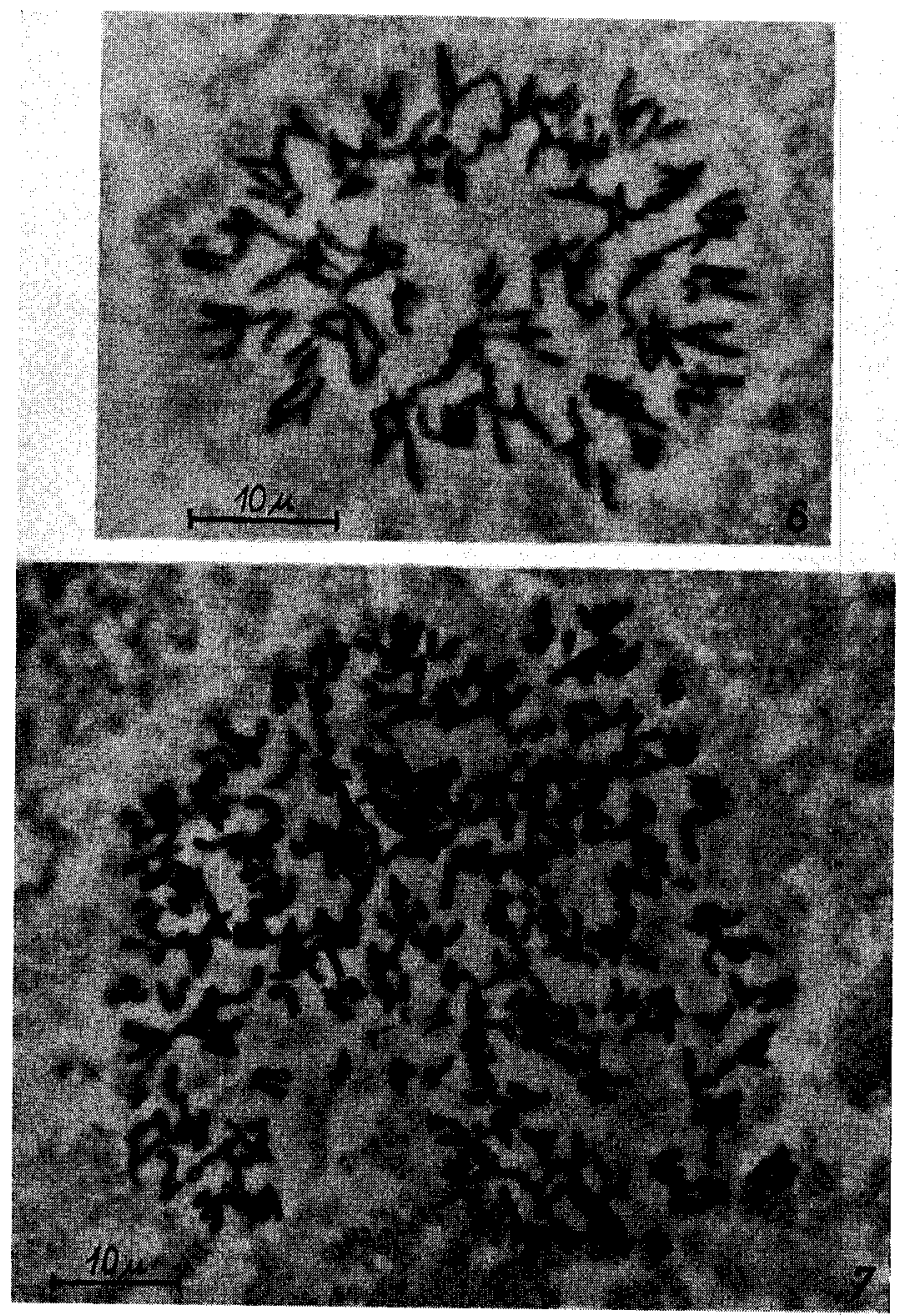

Tafel 2

Fig. 6. Schwebfelchen (Stäfa). Metaphaseplatte mit 146 Chromosomen (siehe dazu Abb. 4) Fig. 7. Albeli (Stäfa). Metaphaseplatte mit $590 \pm 10$ Chromosomen.

(Phasenkontrastaufnahmen) 
nachzuweisen. Dasselbe ist auch in unseren Abbildungen der Fall. Zweiergruppen können hier ziemlich sicher ermittelt werden. Läge hingegen in diesen Kernen eine durch Endomitose hervorgerufene Polyploidie vor, so müssten Gruppen mit mehreren homologen Chromosomen zusammengestellt werden können.

Dass jedoch derartige Fälle trotzdem nicht ausgeschlossen sind, zeigt Abbildung 4, wo eine Metaphaseplatte mit insgesamt 146 Chromosomen analysiert und in Vierergruppen aufgeteilt werden konnte. Dabei sind andeutungsweise zwei untereinander wenig verschiedene Halbsätze erkennbar (zwei Teilgenome?, vgl. S. 227). Auch bei dem in Tabelle I angefuihrten Kern mit $590 \pm$ Io Chromosomen (Tafel 2, Figur 7) ist Polyploidie anzunehmen.

SVAERDSON wies im Zusammenhang mit kleinen Chromosomenzahlen darauf hin, dass diese eventuell durch mehrpolige Anaphase zustande kommen könnten. Wir fanden, wie die verschiedenen Tabellen zeigen, sowohl bei den Chromosomenzahlen (Totalzahlen) als auch bei der Anzahl der phasenverschobenen Chromosomen sowie bei den tripolaren Anaphasen einander entsprechende Zahlen. Es gelang bis jetzt nicht, ein und dieselbe Zahl für sämtliche drei Fälle mit Sicherheit zu ermitteln, doch weist das vorhandene Material darauf hin, dass die kleinen Kerne mit geringer Chromosomenzahl durch Pbasenverschiebung als vorbereitender und durch tripolare Anaphase als ausfübrender Mechanismus zustande kommen können. Wieweit diese Annahme berechtigt ist, werden erst Untersuchungen auf statistischer Basis zeigen.

Die Streuung der Chromosomenzahlen um einzelne mehr oder weniger markante Zahlen (Tabelle I, dazu S. 225) wird wohl auf die von SvaERDSON vielfach beobachtete "non-disjunction» zurückzuführen sein. Wir fanden diese Erscheinung auch gelegentlich in unserem Material, doch musste infolge ungenügender Qualität der betreffenden Stadien auf ihre photographische Wiedergabe verzichtet werden.

Aus den vorgängig skizzierten Resultaten ergibt sich noch eine weitere Konsequenz. Wir sind nämlich - trotz den Angaben von SvaERdSON und KUPKA - neuerdings nicht mehr in der Lage, Cbromosomenzabl pon Coregonus lavaretus L. (Steinmann) anzugeben. Die Svaerdsonschen Zahlen dürfen wir nicht ohne weiteres mit unseren Werten vergleichen, da er mit schwedischen Felchen arbeitete, mit Populationen also, die von denjenigen des schweizerischen Areals geographisch völlig gețrennt sind (siehe dazu SteINManN, I95I). Die Zahlen von KupKa dagegen stimmen mit unseren prinzipiell überein bzw. sind in unserer Liste (Tabelle I) vor- 
handen. Wir halten aber dafür, dass, wennschon eine bestimmte Chromosomenzahl als diploid bezeichnet werden soll, dann am ehesten 84 , da sie weitaus am häufigsten auftritt. Bevor wir jedoch nicht im Besitze genauer zytologischer Untersuchungen über den Chromosomenbestand von Keimzellen oder über die ersten Furchungsteilungen sind, vermögen wir auch keine sicheren Angaben über die haploide oder diploide Chromosomenzahl von Coregonus lavaretus L. (Steinmann) zu machen.

\section{ZUSAMMENFASSUNG}

In der vorliegenden Arbeit werden neue Resultate beschrieben, die nach der Quetschmethode aus Eimaterial von Felchen aus dem Zürichund Untersee gewonnen wurden. Dabei ergeben sich von bisherigen Untersuchungen abweichende Resultate.

So wurde eine ganze Reihe verschiedener Chromosomenzahlen in jeweils ein und demselben Embryo ermittelt. Diese Zahlen stehen untereinander meistens nicht in einem bestimmten Verhältnis, was eine endomitotische Polyploidisierung ausschliesst. Sie können aber, sofern man nur besonders häufig auftretende Zahlen berïcksichtigt, in einer Siebner- oder Zwölferreihe untergebracht werden. Eine Streuung um bestimmte Zahlen herum ist dem Phänomen der «non-disjunction» zuzuschreiben.

Am häufigsten tritt die Zahl 84 auf, deren Satz sich - im Gegensatz $\mathrm{zu}$ Untersuchungen von SVAERDSON und KUPKA - nicht in Vierergruppen unterteilen lässt. Vierergruppen lassen sich dagegen mit einiger Sicherheit an einer analysierten Platte von I46 Chromosomen nachweisen, so dass bei Zahlen über 84 wahrscheinlich durch Endomitose hervorgerufene Polyploidie vorliegt.

Phasenverschiedenheiten im zeitlichen Ablauf der Mitose sowie tripolare Anaphasen deuten auf einen bestimmten Mechanismus der « Kernverkleinerung» hin. Dies um so mehr, als in beiden Phänomenen die gleichen Chromosomenzahlen beteiligt sind, wie sie auch als Totalzahlen kleiner Kerne gefunden werden können.

Es ist heute unmöglich, für Coregonus lavaretus L. (Steinmann) eine bestimmte Chromosomenzahl als haploid zu bezeichnen, da keine diesbezüglichen Spezialuntersuchungen vorhanden sind. Immerhin besteht eine gewisse Wahrscheinlichkeit, dass der Zahl 84 dieser Vorrang zukommt, da sie relativ am häufigsten auftritt. 
Es scheint, dass die erwähnten Abweichungen vom theoretischen Normalfall nicht als Abnormitäten, sondern als für Coregonus lavaretus L. (Steinmann) normal zu betrachten sind.

\section{SUMMARY}

The new results described in the foregoing work were obtained by the smearing technique from the egg material of gwyniads (coregonids) coming from the Lake of Zurich and from the Lake of Constance (near Ermatingen). The results of these experiments deviate from those obtained up to now.

Thus an entire series of different chromosome numbers was discovered, as a rule, in one and the same embryo. In the majority of cases, these numbers do not have a definite relation to one another. This fact excludes an endomitotic polyploidization. However, if we consider only those numbers which come up frequently we can place them in series as multiples of 7 or $\mathrm{I} 2$. A dispersion around certain numbers is due to the phenomenon of non-disjunction.

The number 84 appears most frequently. A set of this number does not show groups of 4 -contrary to the experiments of SVAERDSON and KUPKA. However, groups of 4 can be proven with some certainty on an analysed plate with 146 chromosomes. Consequently, there exists polyploidization through endomitoses in numbers exceeding at least 84 .

Differences in the phases of the successive stages of mitosis as well as tripolar anaphases give indications to a certain mechanism of nucleus decrease, particularly, since in both phenomena the numbers of chromosomes involved can also be found as total chromosome number in smaller nuclei.

Today it is impossible to determine a definite chromosome number as being haploidic for Coregonus lavaretus L. (Steinmann) since no special experiments have been performed up to now.

However, since the number 84 appears most frequently, a certain preeminence can probably be given to it.

It appears that the mentioned deviations from the theoretical normal case are not abnormalities but should be considered as being normal in the development of Coregonus lavaretus L. (Steinmann).

\section{LITERATURVERZEICHNIS}

KUPKA, E., Cbromosomale Verscbiedenbeiten bei schweizerischen Koregonen (Felchen), Rev. suisse Zool. 55 (I948).

KuPKA, E., Die Mitosen- und Cbromosomenverbältnisse bei der grossen Scbmebrenke, Coregonus wartmanni Bloch, des Attersees, Österr. zool. Z. 2, H. s/6 (I950).

STEInManN, P., Monograpbie der schmeizerischen Koregonen, Schweiz. Z. Hydrol. 12, H. I/2;13, H. I (I950/5I).

Svaerdson, G., Chromosome Studies on Salmonidae, Rept. Inst. Freshwater Res. Drottningholm 23 (I945). 